\title{
Harnessing fluid-structure interactions to design self-regulating acoustic metamaterials
}

\section{Citation}

Casadei, Filippo, and Katia Bertoldi. 2014. “Harnessing Fluid-Structure Interactions to Design Self-Regulating Acoustic Metamaterials." Journal of Applied Physics 115 (3) (January 21): 034907.

\section{Published Version}

doi:10.1063/1.4862643

\section{Permanent link}

http://nrs.harvard.edu/urn-3:HUL.InstRepos:12169549

\section{Terms of Use}

This article was downloaded from Harvard University's DASH repository, and is made available under the terms and conditions applicable to Open Access Policy Articles, as set forth at http:// nrs.harvard.edu/urn-3:HUL.InstRepos:dash.current.terms-of-use\#OAP

\section{Share Your Story}

The Harvard community has made this article openly available.

Please share how this access benefits you. Submit a story.

Accessibility 


\section{Harnessing fluid-structure interactions to design self-regulating acoustic metamaterials}

Filippo Casadei ${ }^{1}$ and Katia Bertoldi ${ }^{1,2}$

1) School of Engineering and Applied Sciences, Harvard University, Cambridge MA - USA

2) Kavli Institute for Bionano Science, Harvard University, Cambridge MA - USA

(Dated: 16 December 2013)

The design of phononic crystals and acoustic metamaterials with tunable and adaptive wave properties remains one of the outstanding challenges for the development of next generation acoustic devices. We report on the numerical and experimental demonstration of a locally resonant acoustic metamaterial with dispersion characteristics which autonomously adapt in response to changes of an incident aerodynamic flow. The metamaterial consists of a slender beam featuring a periodic array or airfoil-shaped masses supported by a linear and torsional springs. The resonance characteristics of the airfoils lead to strong attenuation at frequencies defined by the properties of the airfoils and the speed on the incident fluid. The proposed concept expands the ability of existing acoustic bandgap materials to autonomously adapt their dispersion properties through fluid-structure interactions, and has the potential to dramatically impact a variety of applications, such as robotics, civil infrastructures, and defense systems. 


\section{INTRODUCTION}

Metamaterials are engineered micro-structural assemblies that exhibit unique properties not observed in nature or in their constituent materials ${ }^{1}$. An important characteristic of acoustic metamaterials is their ability to tailor the propagation of elastic waves through bandgaps - frequency ranges of strong wave attenuation. Bandgaps are either the result of wave scattering at periodic impedance mismatch zones (Bragg scattering) ${ }^{2}$, or can be generated by resonating units within the medium ${ }^{3}$. While Bragg-type bandgaps have been successfully exploited in phononic crystals to filter, localize and guide acoustic waves ${ }^{4}$, locally resonant metamaterials are capable of generating low frequency attenuation zones ${ }^{3}$ which have been primarily exploited for vibration and noise radiation control applications ${ }^{5,6}$.

Most of the metamaterial configurations proposed so far operate at fixed frequency ranges and it is often impractical, if not impossible, to tune and control their bandgaps after the assembly of the system. In an effort to design tunable materials, it has been shown that Braggtype bandgaps can be controlled through changes in the periodic modulation of impedance mismatch within the medium ${ }^{7-9}$, while in locally resonant metamaterials tuning of functionalities is typically achieved by controlling the natural frequency of the resonating units ${ }^{5,10,11}$. However, these approaches require either a significant amount of energy for actuation which can seriously compromise the major functionality of the structure, or complex hardware architectures which hinder their implementation.

Inspired by the ability of many living organisms, including fishes, insects and bacteria of sensing the surrounding fluid environment to direct their response ${ }^{12-14}$, here we report on a new acoustic metamaterial which exploits fluid-structure interactions to self-regulate its wave propagation characteristics. We consider a locally resonant metamaterial comprising an elastic beam and a periodic array of airfoil-shaped mechanical resonators bonded along its length. The airfoils generate strong attenuation in the beam at frequencies that depend both on their mechanical properties and the speed of the incident fluid flow. Therefore a flow impinging on the system provides the enabling mechanism to alter the bandgaps of the system and achieve unusual wave mechanics. This concept can be considered as an example of an adaptive locally resonant metamaterials capable of self-regulating its dispersion properties in response to variations in the local environment. 


\section{CONCEPT AND MATERIALS}

The considered acoustic metamaterial (Fig. 1a) consists of an aluminum beam of thickness $\mathrm{t}=1.27 \mathrm{~mm}$ and width $\mathrm{w}=2.54 \mathrm{~mm}$ with a periodic arrangement of six airfoil resonators (Fig. 1b) equally-spaced along its length (60 $\mathrm{mm}$ apart). The resonating units comprise a flap
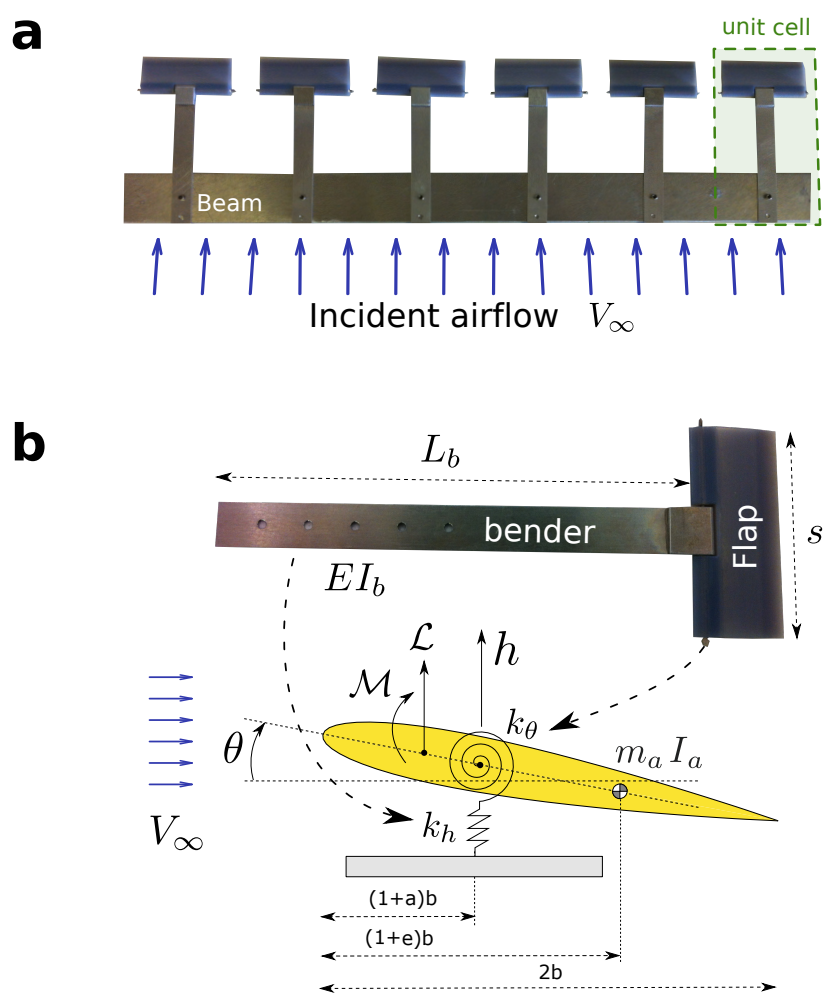

FIG. 1. Harnessing fluid-structure interactions to design self-regulating acoustic metamaterials. a, One-dimensional (1D) metamaterial consisting of an aluminum beam with a periodic array of airfoil resonators bonded along its length. Here, we investigate the effect of the incident air speed $\mathrm{V}_{\infty}$ on the dynamic response of the metamaterial. $\mathbf{b}$, The resonating unit comprises a thin aluminum bender connected to a rigid flap. The unit is modeled as a rigid airfoil with pitch $(\theta)$ and heave/plunge $(h)$ degrees of freedom.

connected to a thin elastic bender. A 3D printer (Objet-500 Connex) is used to fabricate the flaps out of an acrylic-based prototyping material (Young's modulus E 3 GPa and density $\rho=1050 \mathrm{~kg} / \mathrm{m}^{3}$ ) with a NACA 0012 profile. The flap features a rectangular planform with semi chord $b=10 \mathrm{~mm}$ and span $\mathrm{s}=45 \mathrm{~mm}$. Given the modest size of and loads acting on the flaps they are considered as rigid in the following analysis. A $11 \mathrm{~mm}$ wide rectangular cutout is realized at the flap's midspan near the leading edge to host a metallic hinge connecting 
the flap and the bender. The hinge, machined form solid aluminum, allows for rotation of the flap along its span-wise axis (pitch motion), and is connected to the flexible bender using a small amount of superglue (Locktite). The benders are made out of aluminum (Young's modulus $\mathrm{E}=69 \mathrm{GPa}$, and density $\rho=2700 \mathrm{~kg} / \mathrm{m}^{3}$ ) and manually cut from a $0.38 \mathrm{~mm}$ thick plate into strips of width $\mathrm{w}_{b}=10 \mathrm{~mm}$. Five through-thickness holes with diameter $\mathrm{d}=1.0 \mathrm{~mm}$ are realized (10 $\mathrm{mm}$ apart) along its centerline to adjust its bending stiffness. The bender is connected to the main beam by means of a small socket-head hexagonal screw passing through such holes.

\section{AEROELASTIC RESPONSE OF THE RESONATING UNIT}

The coupled aero-elastic behavior of the system, although well understood ${ }^{15}$, is investigated here both numerically and experimentally to provide insights into the self-regulating mechanism of the metamaterial.

We consider a single aerodynamic resonator with mass $m_{a}$ and polar moment of inertia $I_{a}$ which is connected to the primary structure through a flexible bender hinged at the flap's midspan (Fig. 1b). The bender is modeled as a linear spring with elastic constant $k_{h}=3 E I_{b} / L_{b}^{3}, \mathrm{~L}_{b}$ and $E I_{b}$ being the length and bending stiffness of the bender, respectively (Fig. 1b). A torsional spring with stiffness $k_{\theta}$ is introduced to account for any mechanical interference in the hinge. Denoting with $\theta(t)$ and $h(t)$ the pitch and plunge degrees of freedom of the flap (Fig. 1b), the governing equations of the system are obtained as:

$$
\begin{aligned}
& m_{a} \ddot{h}+m_{a} b(e-a) \ddot{\theta}(t)+k_{h} h=\mathcal{L}, \\
& m_{a} b(e-a) \ddot{h}(t)+I_{a} \ddot{\theta}(t)+k_{\theta} \theta(t)=\mathcal{M}+b\left(\frac{1}{2}+a_{f}\right) \mathcal{L},
\end{aligned}
$$

where $b, e$ and $a$ respectively define the semi chord, center of mass and pivoting point of the flap (Fig. 1b). Moreover, $\mathcal{L}$ and $\mathcal{M}$ denote the aerodynamic lift force and moment acting on the airfoil. Here, the classical finite-state induced flow theory by Peters et al. ${ }^{16}$ is used to approximate the unsteady aerodynamic loads of the inviscid, incompressible flow. The 
force and moment acting on the aerodynamic surface are given by

$$
\begin{gathered}
\mathcal{L}=\pi \rho_{\infty} s b^{2}\left(\ddot{h}(t)+V_{\infty} \dot{\theta}(t)-b a \ddot{\theta}(t)\right)+ \\
2 \pi \rho_{\infty} s V_{\infty} b\left[\dot{h}(t)+V_{\infty} \theta(t)+b\left(\frac{1}{2}-a\right) \dot{\theta}(t)-\frac{1}{2} \boldsymbol{b}^{T} \boldsymbol{\lambda}(t)\right], \\
\mathcal{M}=-\pi \rho_{\infty} s b^{3}\left[\frac{1}{2} \ddot{h}(t)+V_{\infty} \dot{\theta}(t)+b\left(\frac{1}{8}-\frac{a}{2}\right) \ddot{\theta}(t)\right],
\end{gathered}
$$

where $\rho_{\infty}=1.225 \mathrm{~kg} / \mathrm{m}^{3}$ is the free stream air density, $s$ the airfoil span, and $\boldsymbol{\lambda}$ is a vector containing the $N_{p}=6$ induced flow terms $\lambda_{n}\left(n=1, \ldots N_{p}\right)$. The evolution of the state vector $\boldsymbol{\lambda}(t)$ is expressed in terms of $N_{p}$ first-order ordinary differential equations as

$$
\boldsymbol{A} \dot{\boldsymbol{\lambda}}(t)+\frac{V_{\infty}}{b} \boldsymbol{\lambda}=\boldsymbol{c}\left[\ddot{h}(t)+V_{\infty} \dot{\theta}(t)+b\left(\frac{1}{2}-a\right) \ddot{\theta}(t)\right]
$$

where $\boldsymbol{A}, \boldsymbol{b}$ and $\boldsymbol{c}$ denote arrays of known coefficients ${ }^{16}$. Substituting Eqs. (2) and (3) into Eq. (1) and introducing the state vector $\boldsymbol{y}(t)=[\boldsymbol{u}(t) \dot{\boldsymbol{u}}(t) \boldsymbol{\lambda}(t)]^{T}$ with $\boldsymbol{u}(t)=[h(t) \theta(t)]^{T}$, the governing equations of the aeroelastic system can be conveniently rewritten in state space form as

$$
\dot{\boldsymbol{y}}(t)=\boldsymbol{\Gamma}\left(V_{\infty}\right) \boldsymbol{y}(t)
$$

The effect of the air speed $V_{\infty}$ on the natural frequencies of the system is investigated calculating the eigenvalues of $\boldsymbol{\Gamma}\left(V_{\infty}\right)$.

Results of this analysis are shown in Fig. 2a,b for airfoils with $\mathrm{L}_{b}=50 \mathrm{~mm}$ (Fig. 2a) and $95 \mathrm{~mm}$ (Fig. 2b). At zero windspeed (i.e. $\mathrm{V}_{\infty}=0$ ) the system features two well distinct natural frequencies associated with the heave and pitch degrees of freedom of the flap. The pitch frequency monotonically increases for increasing values of $\mathrm{V}_{\infty}$, while the opposite trend is observed for the frequency associated with the heave mode. Results also show that after a critical speed ( $\sim 15 \mathrm{~m} / \mathrm{s}$ in Fig. $2 \mathrm{a}$ and $\sim 5 \mathrm{~m} / \mathrm{s}$ in Fig. $2 \mathrm{~b})$ the two resonance frequencies of the airfoil coalescence, generating a dynamic instability of the resonator commonly known as flutter $^{15}$. Above this critical speed the dynamics of the flap becomes highly nonlinear ${ }^{17}$ and the study of this regime falls outside the scope of the present investigation. Finally, comparison of the results obtained for the two bender configurations shows that higher values of $\mathrm{L}_{b}$ increase the compliance of the bender which in turn lowers both the natural frequencies of the flap and its flutter airspeed.

Wind tunnel experiments have also been conducted to validate the predictions of the model. Frequency response measurements of the bender-flap resonator are conducted in a 


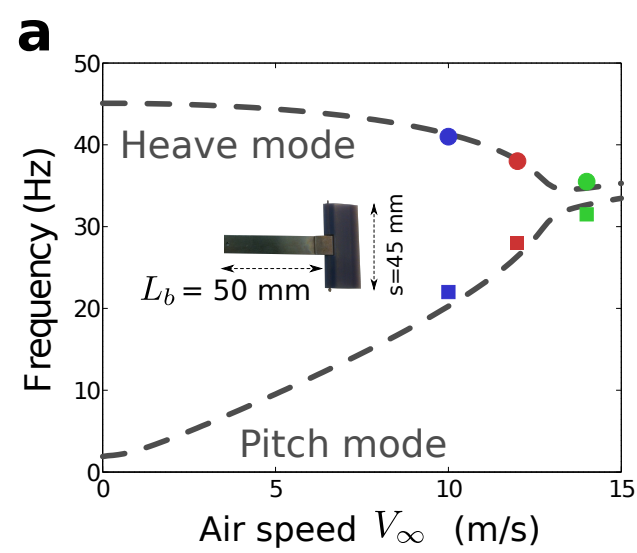

b

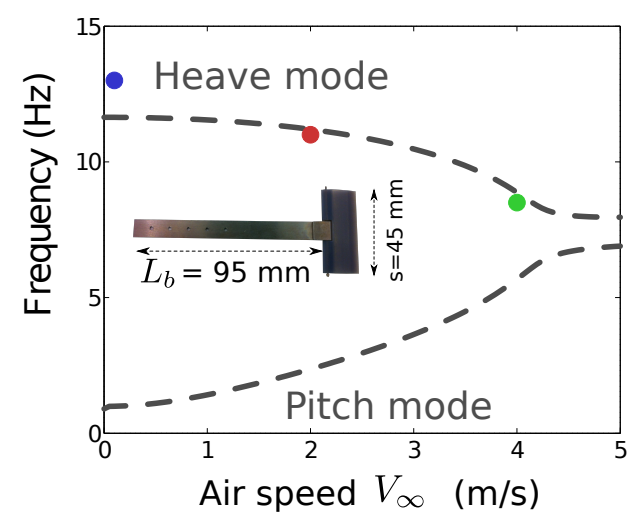

FIG. 2. Numerical and experimental results illustrating the aeroelastic behavior of the resonating unit. a,b, Comparison between the numerical (dashed lines) and experimental (markers) results showing the effect of $\mathrm{V}_{\infty}$ on the natural frequencies of the resonators for two configurations characterized by $\mathrm{L}_{b}=50 \mathrm{~mm}(\mathbf{a})$ and $\mathrm{L}_{b}=95 \mathrm{~mm}(\mathbf{b})$.

low-speed wind tunnel in order to estimate the variation of the system's natural frequencies as a function of the imposed airspeed. Tests are repeated for increasing values of the airspeed form $10 \mathrm{~m} / \mathrm{s}$ to $14 \mathrm{~m} / \mathrm{s}$ for the first sample $\left(\mathrm{L}_{b}=50 \mathrm{~mm}\right)$, and from $0 \mathrm{~m} / \mathrm{s}$ to $4 \mathrm{~m} / \mathrm{s}$ for the second sample $\left(\mathrm{L}_{b}=95 \mathrm{~mm}\right)$. The chosen values of $\mathrm{V}_{\infty}$ coincide with the ones adopted for the transmission test on the finite-size beam (discussed in Section IV). The natural frequencies of the bender-airfoil system, estimated from the resonant peaks of the measured frequency response function, are presented in Fig. 2 showing excellent agreement with the adopted model. Note that for the bender with $\mathrm{L}_{b}=95 \mathrm{~mm}$ measurements can only characterize the highest resonance frequency (associated with the heaving mode), since the adopted experimental apparatus has modest accuracy at frequencies below $5 \mathrm{~Hz}$. 


\section{SELF-REGULATING ACOUSTIC METAMATERIAL}

\section{A. Analysis and testing of the periodic beam}

The aero-elastic behavior of the airfoil units is exploited to generate locally resonant bandgaps in the beam at frequencies which can be controlled through the speed of the incident flow. Finite element analyses are conducted to identify the bandgaps' frequency ranges in the beam and their dependence on $\mathrm{V}_{\infty}$. Analyses are performed through the application of Bloch's theorem ${ }^{18}$ to a unit cell of the periodic assembly comprising a portion of the main beam and one airfoil resonator attached to it (see Fig. 1a). The main beam and the bender are modeled using Euler-Bernoulli beam elements, while Eqs. (1-3) are used to describe the rigid body dynamics of the flap and its interaction with the surrounding fluid. Bloch-Floquet quasi-periodic conditions ${ }^{18,19}$ are applied to the finite element equations of motion of the unit cell ${ }^{20}$ leading to a standard eigenvalue problem which is solved for the complex propagation constant $\mu(\omega)$ of the system ${ }^{20}$. The real part of $\mu$, known as attenuation constant, denotes the rate of exponential decay that a wave experiences as it propagates through the medium. Wave propagation is therefore possible within frequency bands where $\mu$ is purely imaginary, while bandgaps occur at frequencies characterized by a non zero attenuation constant.

The tunable properties of the metamaterial are also verified experimentally through wave transmission tests performed on the finite-size sample shown in Fig. 1. The sample is positioned inside the test section of a low-speed wind tunnel (Engineering Laboratory Design Inc.) which is manually set to operate at a given airspeed. The beam is clamped at one end and free to vibrate at the opposite end (see Fig. 3a). An electrodynamic shaker (model K2025E013 from The Modal Shop) provides a random (broadband) input to the beam through a load cell (208C01 PCB Piezotronics) located near the clamp. The dynamic response of the metamaterial is measured in terms of a transmission coefficient defined as the ratio between the acceleration signals measured at the two ends of the beam using two identical accelerometers (352C22 PCB Piezotronics). Measurements are recorded using a NI-cDAQ-9174 acquisition system and related software. 


\section{B. Results and discussion}

Numerical and experimental results for the case with $\mathrm{L}_{b}=50 \mathrm{~mm}$ are reported in Figs. $3 \mathrm{~b}$ and c showing the evolution of the attenuation constant and transmission coefficient as a function of the frequency of wave propagation for different wind speeds $\mathrm{V}_{\infty}=10,12,14 \mathrm{~m} / \mathrm{s}$. Results
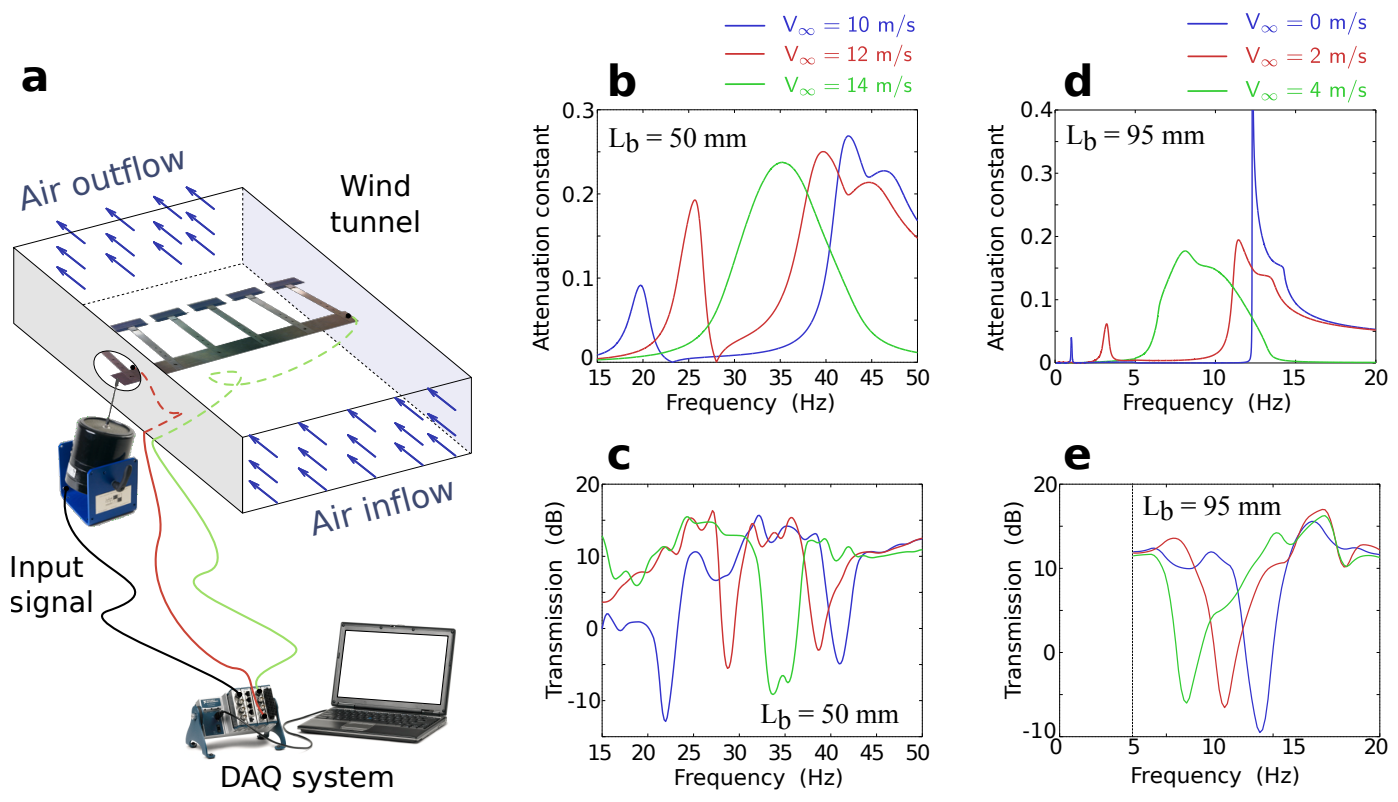

FIG. 3. Self-regulating attenuation properties. a, Schematic of the experimental setup used to characterize the dynamic response of the system. $\mathbf{b}$ and $\mathbf{d}$, Attenuation constant of the beam computed for $\mathrm{L}_{b}=50 \mathrm{~mm}(\mathbf{b})$ and $\mathrm{L}_{b}=95 \mathrm{~mm}(\mathbf{d})$. Peaks of the attenuation constant identify frequency regions of strong wave attenuation (bandgaps) which occur in the vicinity of the pitch and plunge resonance frequencies of the corresponding airfoils shown in Fig. 2a,b. Results computed for different flow velocities show that the bandgaps tend to coalesce as $\mathrm{V}_{\infty}$ increases, and that they fully coalesce at the flutter speed. $\mathbf{c}$ and e, Transmission coefficient measured on the finite size beam with six airfoil resonators attached along its length. The presence of bandgaps is indicated by frequency regions where the transmission coefficient suddenly drops (valleys) about $15 d B$. The experimental results $(\mathbf{c}, \mathbf{e})$ are in excellent agreement with the trend predicted by FE analysis $(\mathbf{b}, \mathbf{d})$.

indicate that the system features two distinct frequency bandgaps, identified by peaks of the attenuation constant and corresponding valleys of the transmission coefficient. These occur in the vicinity of the pitch and plunge resonance frequencies of the corresponding 
airfoils shown in Fig. 2a, confirming the locally-resonance nature of the attenuation regions. Remarkably, because of the modal behavior of the resonating unit, an increase in $\mathrm{V}_{\infty}$ results in a shift of the bandgaps frequencies, which gradually approach each other. These results clearly demonstrate the self-regulating properties of the structure whose bandgaps autonomously adapt to different flow speeds. The analysis is conducted up to a maximum speed $\mathrm{V}_{\infty}=14 \mathrm{~m} / \mathrm{s}$ after which the model predicts the onset of a flutter instability of the airfoils. Interestingly, for this value of $\mathrm{V}_{\infty}$ the two bandgaps of the system merge in a single broad attenuation region centered at $\sim 35 \mathrm{~Hz}$ (see Fig.3b).

To demonstrate the robustness of the proposed concept, in Figs. 3d and e we report the results corresponding to a longer bender $\left(L_{b}=95 \mathrm{~mm}\right)$. As shown in Fig. 3d, a more flexible connection between the airfoils and the main beam lowers the airfoils resonance frequencies between to $2 \mathrm{~Hz}$ and $10 \mathrm{~Hz}$. Because of the modest accuracy of the experimental apparatus at frequencies below $5 \mathrm{~Hz}$, the experimental results presented in Fig. 3e only capture the highest bandgap associated with the heaving mode of the airfoil. It is also worth noting the excellent agreement between the numerical results and the experimentally identified bandgaps occurring at frequencies centered in the vicinity of the attenuation peaks predicted by the numerical analysis, which also coincide with the natural frequencies associated with the pitch and plunge motion of the airfoils (Fig.2b).

Although this study is conducted on a one-dimensional configuration realized at the centimeter scale, the proposed concept has the potential to be extended to arbitrary twoand three- dimensional configurations and to a broader range of length scales. In particular, in Fig. 4 we investigate numerically the relationship between the resonance frequency of the airfoils, their overall length scale and the incident airspeed $V_{\infty}$. Since the aerodynamic model adopted in this study assumes an inviscid and irrotational flow ${ }^{16}$, our analysis correctly captures the response of systems characterized by Reynolds numbers $(R e)$ approximately greater than 100 (i.e. $R e>10^{2}$ ). Remarkably, our results indicate that the flow regime remains inviscid and irrotational even when the overall size of the system is reduced at the sub-millimeter length scale, although this will inevitably affect its frequency range of operation. 


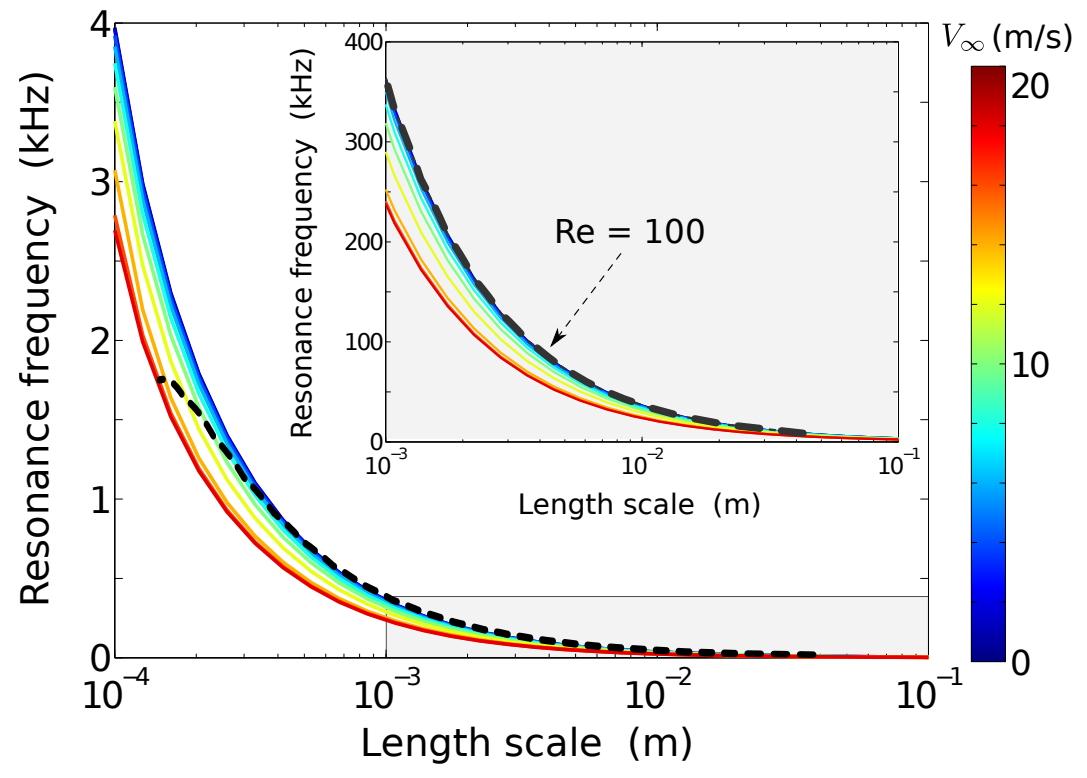

FIG. 4. Scaling properties of the proposed concept. Numerical results indicating the relationship between the first natural frequency of a resonating unit, the overall length scale of the resonator, and the magnitude of the incident air speed $\mathrm{V}_{\infty}$. The thick-dashed line represents configurations characterized by $\operatorname{Re}>10^{2}$. Below this line the flow regime can be considered as inviscid and irrotational, so that the elastic and aerodynamic behavior of the airfoil resonators is accurately predicted by the present model, regardless of the specific scale of the system.

\section{CONCLUDING REMARKS}

We have investigated fluid-structure interactions in locally resonant materials and shown how they can be exploited to design structures with self-regulating dispersion properties. Our results demonstrate that the airspeed impinging on the system provides an effective mechanism to autonomously alter its bandgaps frequency ranges without resorting to additional stimuli. This intriguing dynamic behavior is enabled by the use of airfoil-type resonating units that behave as aeroelastic systems subjected to modal convergence. A combination of numerical and experimental analyses is used to illustrate the concept and to gain insights into the correlation between the aeroelastic behavior of the resonators and the dynamic properties of the periodic system.

By harnessing fluid-structure interactions, we expand the capabilities of existing acoustic bandgap materials and design systems capable of sensing the surrounding environment and change their dynamic response accordingly. This concept has the potential to dramat- 
ically impact a variety of applications, such as robotics, civil infrastructures, and defense systems. For example, the proposed mechanism can lead to the design of sustainable and self-regulating vibration suppression devices capable of autonomously tracking and controlling the dynamic response of structures over a broad range of operative conditions. Furthermore, the growing opportunities offered by novel micro-fabrication techniques allows its integration with other micro-scale devices leading to complex hierarchical systems capable

of self-responding to local environmental variations. Finally, the possibility of extending the concept to two- and three-dimensional configurations, promises the development of novel self-adaptive coatings for stress wave management applications.

\section{ACKNOWLEDGMENTS}

This work has been partially supported by the Wyss Institute through the Seed Grant Program. K.B. acknowledges startup funds from the School of Engineering and Applied Sciences, Harvard. F.C. and K.B. are also grateful to Michael Smith and Prof. Robert J. Wood for their support with the wind tunnel experiments.

\section{REFERENCES}

${ }^{1}$ J. Pendry, a.J. Holden, D. Robbins, and W. Stewart, "Magnetism from conductors and enhanced nonlinear phenomena," IEEE Transactions on Microwave Theory and Techniques 47, 2075-2084 (1999).

${ }^{2}$ C. Kittel, "Introduction to solid state physics," American Journal of Physics 35, 547-548 (1967).

${ }^{3}$ Z. Liu, X. Zhang, Y. Mao, Y. Zhu, Z. Yang, C. Chan, and P. Sheng, "Locally resonant sonic materials," Science 289, 1734-1736 (2000).

${ }^{4}$ F. Hsiao, A. Khelif, H. Moubchir, A. Choujaa, C. Chen, and V. Laude, "Waveguiding inside the complete band gap of a phononic crystal slab," Physical Review E 76, 056601 (2007).

${ }^{5}$ F. Casadei, M. Ruzzene, L. Dozio, and K. Cunefare, "Broadband vibration control through periodic arrays of resonant shunts: experimental investigation on plates," Smart materials and structures 19, 015002 (2009). 
${ }^{6}$ F. Casadei, L. Dozio, M. Ruzzene, and K. Cunefare, "Periodic shunted arrays for the control of noise radiation in an enclosure," Journal of Sound and Vibration 329, 36323646 (2010).

${ }^{7} \mathrm{~K}$. Bertoldi and M. Boyce, "Wave propagation and instabilities in monolithic and periodically structured elastomeric materials undergoing large deformations," Physical Review B 78, 184107 (2008).

${ }^{8}$ N. Boechler, J. Yang, G. Theocharis, P. Kevrekidis, and C. Daraio, "Tunable vibrational band gaps in one-dimensional diatomic granular crystals with three-particle unit cells," Journal of Applied Physics 109 (2011).

${ }^{9}$ Y. Yao, Z. Hou, and Y. Liu, "The two-dimensional phononic band gaps tuned by the position of the additional rod," Physics Letters A 362, 494-499 (2007).

${ }^{10}$ F. Casadei, T. Delpero, A. Bergamini, P. Ermanni, and M. Ruzzene, "Piezoelectric resonator arrays for tunable acoustic waveguides and metamaterials," Journal of Applied Physics 112, 064902-064902 (2012).

${ }^{11}$ I. Shadrivov, S. Morrison, and Y. Kivshar, "Tunable split-ring resonators for nonlinear negative-index metamaterials," arXiv preprint physics/0608044 (2006).

${ }^{12}$ H. C. Fu, T. R. Powers, R. Stocker, et al., "Bacterial rheotaxis," Proceedings of the National Academy of Sciences 109, 4780-4785 (2012).

${ }^{13}$ J. C. Montgomery, C. F. Baker, and A. G. Carton, "The lateral line can mediate rheotaxis in fish," Nature 389, 960-963 (1997).

${ }^{14}$ D. A. Lytle, J. D. Olden, and L. E. McMullen, "Drought-escape behaviors of aquatic insects may be adaptations to highly variable flow regimes characteristic of desert rivers," The Southwestern Naturalist 53, 399-402 (2008).

${ }^{15}$ A. Collar, "The first fifty years of aeroelasticity," Aerospace 5, 12-20 (1978).

${ }^{16}$ D. A. Peters, S. Karunamoorthy, and W.-M. Cao, "Finite state induced flow models. i-two-dimensional thin airfoil," Journal of Aircraft 32, 313-322 (1995).

${ }^{17}$ I. Garrick and W. H. Reed III, "Historical development of aircraft flutter," Journal of Aircraft 18, 897-912 (1981).

${ }^{18} \mathrm{~L}$. Brillouin, Wave propagation in periodic structures: electric filters and crystal lattices (Courier Dover Publications, 2003).

${ }^{19} \mathrm{~F}$. Bloch, "Über die quantenmechanik der elektronen in kristallgittern," Zeitschrift für physik 52, 555-600 (1929). 
${ }^{20}$ D. J. Mead, "Wave propagation in continuous periodic structures: research contributions from southampton, 1964-1995," Journal of Sound and Vibration 190, 495-524 (1996). 